Notiz betr. Komet 1904 a.

(Auszug aus einem Briefe an den Herausgeber).

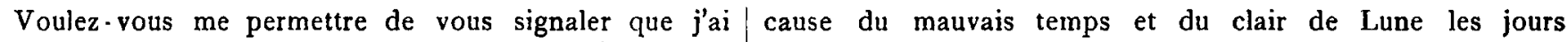
découvert la comète r 904 a sur un cliché que j'ai pris le suivants. 16 avril de $10^{\mathrm{h}} 15^{\mathrm{m}}$ à $10^{\mathrm{h}} 45^{\mathrm{m}}$ t. $\mathrm{m}$. de Paris.

Sur ce cliché obtenu avec un objectif à portraits de $4 \mathrm{~cm}$ d'ouverture, j'avais remarqué un petit point nébuleux sans pouvoir en faire par la suite l'identification, à

J'ai appris seulement le 26 avril la découverte de la comète Brooks, faite en Amérique, presque simultanément, à la position de l'objet que j'avais trouvé photographiquement... $\left.{ }^{*}\right)$

Observatoire privé, Donville (Manche), 1904 Avril 27.

Lucien Rudaux.

*) Ein dem Briefe beiliegender Abzug der Platte zeigt den Kometen mit aller wünschenswerten Deutlichkeit. $K r$.

\title{
Comet 1904 a.
}

(Astronomical Bulletin of the Harvard College Observatory).

The following observations of the positions of Brooks' comet include those that have been received at the Harvard College Observatory, and those obtained here.*)

\begin{tabular}{|c|c|c|c|c|}
\hline $\begin{array}{c}1904 \\
\text { Gr. M. T. }\end{array}$ & $\alpha$ & 8 & & Authority \\
\hline pril 2.826 & $\mathrm{r} 7^{\mathrm{h}} 25^{\mathrm{m}} 30^{\mathrm{s}}$ & $+35^{\circ} 3^{\prime}$ & & H. C. O. \\
\hline I 6.870 & I $65^{6} 32$ & +4422 & & 》 \\
\hline I 7.552 & 1655 & +4448 & & Brooks \\
\hline I 7.6579 & 165550.18 & +4451 & 30.1 & Naval Obs. \\
\hline I $7.666 \mathrm{I}$ & I6 $55 \quad 48.87$ & +4451 & 50.1 & $»$ \\
\hline I 7.8717 & I 655 I 4.5 I & +4459 & 37.6 & 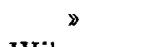 \\
\hline $\mathrm{I} 8.689 \mathrm{I}$ & I6 $52 \quad 57.5$ & $+453^{\circ}$ & I 3 & Wilson \\
\hline
\end{tabular}

Cambridge Mass., 1904 April 20.
The positions marked H.C.O., including six before the comet was discovered, are derived from the Harvard photographs. The positions are for 1855 . They are only approximate and depend on BD. places of the stars.

Two photographs taken on April 16, with an objective prism, show a nearly continuous spectrum with a slight increase in light at two points, so that the distribution of light seems to be the same as in a similar photograph of Comet I 898 VII.

*) Die bereits telegraphisch mitgeteilten und in Nr. 3941 und 3943 veröffentlichten Positionen H.C.O. März I1, 15, April I, 5, 13, 16.855, Brooks April 16, Seares April 17 und 18, Aitken April 17 sind hier weggelassen worden. Kr.

\section{Observations de la comète 1904 a}

faites à Nice (grand équatorial de $0^{\mathrm{m}}{ }^{7} 6$ d'ouverture) par M. St. Favelle.

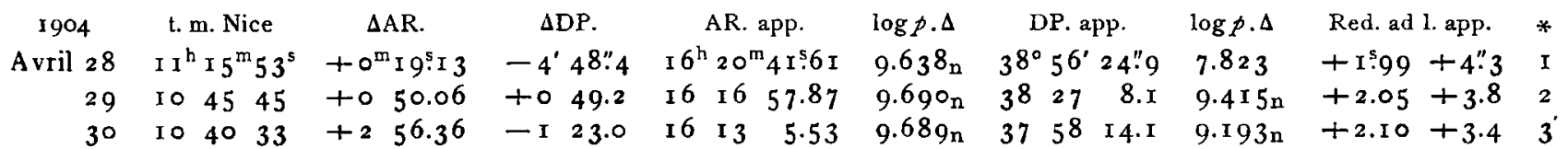

Etoiles de comparaison pour 1904.0.

\begin{tabular}{|c|c|c|c|c|c|c|c|c|c|}
\hline & \multicolumn{2}{|c|}{ AR. } & & \multicolumn{2}{|c|}{ DP. } & 0 & \multicolumn{3}{|c|}{$\begin{array}{l}\text { Autorité } \\
\text { AG. Cambr. } 4985\end{array}$} \\
\hline & I 6 & I 6 & 5.76 & 38 & 26 & $\begin{array}{r}7.1 \\
\end{array}$ & ๖ & 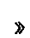 & 496 \\
\hline & 16 & IO & 7.07 & 37 & 59 & 33.7 & 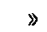 & 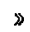 & \\
\hline
\end{tabular}

1904 NN. Die in Nr. 3941 p. 78 mitgeteilte Beobachtung von April 44 muß in Dekl. lauten: + $8^{\circ} 10^{\prime} 5^{\prime \prime}$. . F. Palisa.

Berichtigungen zu Nr. 3399 Bd. 142 p. 230 Z. I 5 v. u. statt: Seite 35 lies: Seite 55.

$\gg, \gg$, Z. IO v. u. statt: +o."20 $(t-1875)$ lies: $+0.02(t-1875)$.

» 3938 \$ $165 \gg 19$ Z.17 v. o. statt: $0.0001103 \tau^{4}$ lies: $0.0001903 \tau^{4}$.

Inhalt zu Nr. 3946. W. Luther. Ringmikrometer-Beobachtungen von kleinen Planeten. 145. - A. Prey. Bestimmung des Massenverhältnisses bei 70 Ophiuchi. 153. - W. Ceraski. Une nouvelle variable 18.1904 Ophiuchi. 157. - Th. D. Anderson. New variable star 19. I904 Leonis minoris. I57. - Beobachtungen des Planeten I904 NY. I 57. - L. Rudaux. Notiz betr. Komet I904a. 159. - E.C. Pickering. Comet 1904 a. - St. Favelle. Observations de la comète 1904 a. 159. - F. Palisa. Planet 1904 NN. 159. - Berichtigungen. I 59. 